\title{
Analysis of recent shared ancestry in a familial cohort identifies coding and noncoding autism spectrum disorder variants
}

\author{
Islam Oguz Tuncay (D) ${ }^{1}$, Nancy L. Parmalee ${ }^{2}$, Raida Khali ${ }^{3}$, Kiran Kaur ${ }^{2}$, Ashwani Kumar ${ }^{2}$, Mohamed Jimale ${ }^{2}$, Jennifer L. Howe ${ }^{4}$, \\ Kimberly Goodspeed ${ }^{5,6,7}$, Patricia Evans ${ }^{5,6,7}$, Loai Alzghoul ${ }^{8}$, Chao Xing $\mathbb{D}^{2,9,10}$, Stephen W. Scherer (D) $^{4,11}$ and \\ Maria H. Chahrour (iD) $1,2,7,12,13 \otimes$
}

\begin{abstract}
Autism spectrum disorder (ASD) is a collection of neurodevelopmental disorders characterized by deficits in social communication and restricted, repetitive patterns of behavior or interests. ASD is highly heritable, but genetically and phenotypically heterogeneous, reducing the power to identify causative genes. We performed whole genome sequencing (WGS) in an ASD cohort of 68 individuals from 22 families enriched for recent shared ancestry. We identified an average of 3.07 million variants per genome, of which an average of 112,512 were rare. We mapped runs of homozygosity (ROHs) in affected individuals and found an average genomic homozygosity of $9.65 \%$, consistent with expectations for multiple generations of consanguineous unions. We identified potentially pathogenic rare exonic or splice site variants in 12 known (including KMT2C, SCN1A, SPTBN1, SYNE1, ZNF292) and 12 candidate (including CHD5, GRB10, PPP1R13B) ASD genes. Furthermore, we annotated noncoding variants in ROHs with brainspecific regulatory elements and identified putative disease-causing variants within brain-specific promoters and enhancers for 5 known ASD and neurodevelopmental disease genes (ACTG1, AUTS2, CTNND2, CNTNAP4, SPTBN4). We also identified copy number variants in two known ASD and neurodevelopmental disease loci in two affected individuals. In total we identified potentially etiological variants in known ASD or neurodevelopmental disease genes for $\sim 61 \%$ (14/23) of affected individuals. We combined WGS with homozygosity mapping and regulatory element annotations to identify candidate ASD variants. Our analyses add to the growing number of ASD genes and variants and emphasize the importance of leveraging recent shared ancestry to map disease variants in complex neurodevelopmental disorders.
\end{abstract}

npj Genomic Medicine (2022)7:13; https://doi.org/10.1038/s41525-022-00284-2

\section{INTRODUCTION}

Autism spectrum disorder (ASD) is a neurodevelopmental condition with a current population prevalence estimated at 1 in 44 individuals in the USA ${ }^{1}$. Growing evidence suggests that ASD encompasses a collection of individually rare disorders that share the core features of difficulty with social and communication skills and stereotyped or repetitive behaviors and interests ${ }^{2}$. ASD varies in the severity and presentation of the core symptoms as well as the associated comorbidities, which include intellectual disability and seizures among others. ASD is primarily genetic, with heritability estimates ranging from 83 to $91 \%^{3-5}$. Studies to date have identified hundreds of ASD genes, with causative de novo and inherited variants of differing effect sizes and frequencies, with approximately 100 of those genes presumed to harbor highly penetrant variants ${ }^{6,7}$. Additionally, risk-conferring copy number variants (CNVs) have been identified, highlighting the complex genetic architecture of $\mathrm{ASD}^{8-15}$. Each of the currently known ASD genes accounts for less than $1 \%$ of cases $^{7}$, and all the rare variants identified to date account for only $\sim 30 \%$ of the disease burden, in particular when considering families with complex phenotypes ${ }^{16}$.
Rare biallelic events are estimated to contribute to $5 \%$ of the ASD burden of disease, with that percentage increasing to $10 \%$ in females ${ }^{17}$. Variant discovery of rare alleles of large effect requires sequencing of large numbers of individuals, and for sufficiently rare alleles, it is unlikely that they will be observed in a homozygous state in a nonconsanguineous population. Previous studies have successfully analyzed runs of homozygosity in consanguineous families to discover rare variants contributing to recessive disease ${ }^{18-22}$. This has proved to be an effective strategy to identify genes that were previously not known to be involved in $\mathrm{ASD}^{18,23-25}$, contributing to the understanding of the underlying biology of the disorder.

In this study, we ascertained a familial cohort through probands with ASD and aimed to utilize the recent shared ancestry within the cohort to detect rare and ultra-rare pathogenic biallelic variants, which would be difficult to identify in nonconsanguineous cohorts. This unique collection of ancestrally diverse families had representation from the Middle East (47 individuals), South Asia (12 individuals), and Europe (5 individuals), in addition to one East Asian individual, one Hispanic individual, one individual of mixed European and East Asian ancestry, and one

\footnotetext{
'Department of Neuroscience, University of Texas Southwestern Medical Center, Dallas, TX 75390, USA. ${ }^{2}$ Eugene McDermott Center for Human Growth and Development University of Texas Southwestern Medical Center, Dallas, TX 75390, USA. ${ }^{3}$ Department of Biotechnology and Genetic Engineering, Faculty of Science, University of Philadelphia, Amman, Jordan. ${ }^{4}$ The Centre for Applied Genomics and Program in Genetics and Genomic Biology, Hospital for Sick Children, Toronto, ON, Canada. ${ }^{5}$ Department of Pediatrics, University of Texas Southwestern Medical Center, Dallas, TX 75390, USA. ${ }^{6}$ Department of Neurology, University of Texas Southwestern Medical Center, Dallas, TX 75390, USA. ${ }^{7}$ Department of Psychiatry, University of Texas Southwestern Medical Center, Dallas, TX 75390, USA. ${ }^{8}$ Department of Physiology and Biochemistry, School of Medicine, The University of Jordan, Amman, Jordan. ${ }^{9}$ Department of Population and Data Sciences, University of Texas Southwestern Medical Center, Dallas, TX 75390 , USA. ${ }^{10}$ Lyda Hill Department of Bioinformatics, University of Texas Southwestern Medical Center, Dallas, TX 75390, USA. ${ }^{11}$ McLaughlin Centre and Department of Molecular Genetics, University of Toronto, Toronto, ON, Canada. ${ }^{12}$ Center for the Genetics of Host Defense, University of Texas Southwestern Medical Center, Dallas, TX 75390 , USA. ${ }^{13}$ Peter O'Donnell Jr. Brain Institute, University of Texas Southwestern Medical Center, Dallas, TX 75390, USA. ${ }^{\circledR}$ email: maria.chahrour@utsouthwestern.edu
} 
individual of mixed European and Hispanic ancestry. We performed whole genome sequencing (WGS) followed by homozygosity mapping and mining of regulatory element annotations, and focused on rare deleterious variants as candidate diseasecausing. We identified 24 genes with 34 nonsynonymous exonic or splice site variants in 18 affected individuals. Of the identified genes, 12 have been previously implicated in ASD and other neurodevelopmental disorders and 12 are new candidate ASD genes expressed in the brain and characterized as having neurodevelopmental functions with potential consequences in disease. For 2 affected individuals, we identified CNVs overlapping with known ASD or neurodevelopmental disease loci. In addition, we identified 37 inherited homozygous variants within brainspecific regulatory elements, 5 of which were located within promoters or enhancers for known ASD genes. Overall, we identified potentially etiological variants in known ASD or neurodevelopmental disease genes for 14 out of 23 affected individuals. Biallelic events involving rare and ultra-rare variants seldom occur in nonconsanguineous populations. By leveraging the enriched homozygosity in this consanguineous cohort, we were able to identify biological processes and mechanisms that will generalize to ASD in other populations.

\section{RESULTS}

\section{Clinical characteristics of the ASD consanguineous cohort}

A total of 22 families, including 20 trios, 1 quad with two affected siblings, and 1 quad with an affected proband and his unaffected fraternal twin, were enrolled in our study (see Supplementary Table 1). The majority of families $(68 \%, 15 / 22)$ reported consanguinity through first-cousin unions. The cohort comprised a total of 23 affected individuals and their family members, with an affected male to female ratio of 6.7 (20 males, 3 females). Language and speech impairments were identified in all affected individuals tested $(N=16)$ and $56 \%$ of them were non-verbal. Other notable phenotypes included intellectual disability (5/9 affected individuals assessed), developmental delay (8/8), attention deficit hyperactivity disorder (4/8), and seizures (1/18) (see Table 1). All affected individuals tested had normal brain imaging on MRI and CT scans $(N=11)$.

\begin{tabular}{|lll|}
\hline $\begin{array}{l}\text { Table 1. Demographics and clinical information for the } \\
\text { consanguineous ASD cohort. }\end{array}$ & \\
\hline Clinical symptoms in & $\begin{array}{l}\text { Probands } \\
\text { assessed }(N)\end{array}$ & $\begin{array}{l}\text { Probands with } \\
\text { phenotype }(N)\end{array}$ \\
probands & 23 & 23 \\
\hline ASD & 16 & 16 \\
Speech impairment & & 7 \\
$\quad$ Verbal & & 9 \\
$\quad$ Non-verbal & 9 & 5 \\
Intellectual disability & 8 & 8 \\
Developmental delay & 18 & 1 \\
Seizures & 8 & 4 \\
Learning disabilities & 8 & 4 \\
Attention deficit hyperactivity & & \\
disorder & 8 & 4 \\
Gastrointestinal problems & & Average (Range, years) \\
\hline & & $2.7(0.75-7.0)$ \\
\hline & $33.7(24.3-45.7)$ \\
\hline Age at diagnosis $(N=20)$ & $31.0(24.0-39.6)$ \\
Paternal age at birth $(N=10)$ & & \\
Maternal age at birth $(N=10)$ & &
\end{tabular}

The majority of families in our cohort (15/22) are of Jordanian descent, including 13 families recruited in Jordan and 2 families recruited in the United States (see Supplementary Table 1). We used principal component analysis (PCA) to explore the ancestry of the families in the cohort. The majority of samples in the cohort clustered adjacent to European samples from the 1000 Genomes project $(1000 \mathrm{G})^{26}$ with separation from other European subpopulations. In addition to individuals of Jordanian ancestry, our cohort includes individuals with ancestry from the Asian Subcontinent and a family from Peru. Samples from these families clustered as expected with the $1000 \mathrm{G}$ subpopulations from the corresponding regions (see Supplementary Fig. 1).

\section{Whole genome sequencing and variant discovery}

We performed WGS on samples from 68 individuals including 23 affected children. The average read depth was $37 X$, with no differences in depth of sequencing with respect to affection status, sex, or family relationships (see Supplementary Figs. 2a-c). On average, $99.6 \%$ and $95.6 \%$ of bases were covered at a mean read depth of at least $10 \times$ and $20 \times$, respectively (see Supplementary Fig. 2d).

An average of $4,819,156$ total variants were identified per genome. After applying read depth and quality filters, 3,071,060 variants per genome remained, of which an average of 2,666,208 were single nucleotide variants (SNVs) and 404,852 were insertions or deletions (indels) (see Supplementary Table 2). A detailed summary of our WGS data processing and variant filtration pipeline is shown in Supplementary Fig. 3. We filtered for rare variants with a minor allele frequency (MAF) $<1 \%$ in all annotated population databases (1000G ${ }^{26}$, Genome Aggregation Database $(\text { gnomAD })^{27}$, and Greater Middle East Variome Project $\left.(\mathrm{GME})^{28}\right)$, identifying on average 112,512 rare variants per genome, of which 110,450 were heterozygous and 2,063 were homozygous (see Supplementary Table 2). Affection status, sex, or family relationships had no significant effect on the average variant counts in any category (see Supplementary Fig. 4). We discovered an average of 34,840 ultra-rare variants per genome, and a total of $1,193,026$ unique ultra-rare variants in the cohort that have not been reported in any of the public databases that we used for annotation (see Supplementary Table 2). Out of these variants an average of 5,580 were private (8567 for parents, 105 for offspring), meaning present only in a single individual in the cohort. We identified an average of 24 (36 for parents, 1 for offspring) private exonic or splice site (referred to as coding) variants per genome, of which 8 (13 for parents, 0.4 for offspring) per genome were nonsynonymous and predicted to be deleterious (see Supplementary Table 2).

To assess whether there was an excess of potentially pathogenic variants in affected compared to unaffected individuals, we performed a burden analysis. We found no difference between affected and unaffected individuals in the burden of rare variants with nondisrupting (ND), missense damaging (MD), or loss of function (LoF) effects (see Supplementary Fig. 5). This was expected due to the consanguinity in our cohort, the small sample size, and the lack of unaffected siblings (except for one family with one unaffected sibling).

\section{Analysis of copy number variation}

We identified copy number aberrations in affected individuals by using CNVkit ${ }^{29}$. Briefly, average read depth in affected individuals was compared to the average read depth in unaffected family members across the genome. We identified an average of 310 genomic regions with copy number aberrations per affected individual, ranging in size from $2.2 \mathrm{~Kb}$ to $93.6 \mathrm{Mb}$. Out of the identified CNVs, a total of 1790 overlapped with known ASD CNVs reported in the CNV Module of the SFARI Gene database ${ }^{6}$, and were designated as "overlapping". To further focus on potentially 
clinically relevant CNVs, we filtered for "overlapping" CNVs that were larger than the median size of the corresponding SFARI CNV in ASD cases, and we identified 22 such CNVs in 10 affected individuals (see Supplementary Table 3). To identify genomic regions that were significantly deleted or amplified across all affected individuals, we analyzed the output from CNVkit ${ }^{29}$ with GISTIC2.0 $0^{30}$. We identified 113 significant regions (62 amplifications and 51 deletions), of which 19 (10 amplifications and 9 deletions) overlapped with known ASD CNVs. We then filtered for CNVs that were larger than the median size of the corresponding SFARI CNV, and we identified 4 such CNVs, 2 amplifications and 2 deletions (see Supplementary Table 4).

\section{Homozygosity analysis}

Consanguineous populations have higher proportions of genomic homozygosity. Recent shared ancestry results in larger genomic blocks that are inherited identical by descent as compared to outbred populations ${ }^{31}$. Rare homozygous variants are therefore likely to be present in runs of homozygosity $(\mathrm{ROH})$ in the offspring of consanguineous unions ${ }^{32}$. Due to the consanguinity in our cohort, we utilized homozygosity analysis to identify regions of the genome that are homozygous and likely to harbor rare recessive mutations.

We identified an average of 183 autosomal runs of homozygosity (ROHs) per genome that are each $>1 \mathrm{Mb}$ in size. The average $\mathrm{ROH}$ length was $1.52 \mathrm{Mb}$ per genome (see Fig. 1a), with the largest $\mathrm{ROH}$ in each genome ranging from $3 \mathrm{Mb}$ to $7.6 \mathrm{Mb}$. The total length of ROHs averaged $278.7 \mathrm{Mb}$ (see Supplementary Fig. 6a) corresponding to $9.65 \%$ of the genome (see Fig. 1b). Since the expected homozygosity in genomes of offspring from first cousin and double-first cousin unions is $6.25 \%$ and $12.5 \%^{31}$, respectively, our results suggested a second to third degree relationship between parents in our cohort, in line with self-reported information from study participants. Apart from a minor increase in average $\mathrm{ROH}$ size in males, we saw no difference in $\mathrm{ROH}$ metrics in correlation with affection status, sex, or family relationships (see Fig. 1a and b, and Supplementary Fig. 6).

In order to identify shared $\mathrm{ROH}$ segments between affected individuals, we combined all $\mathrm{ROH}$ s that were found in at least 2 affected individuals to construct 448 merged $\mathrm{ROH}$ segments ranging in size from $1 \mathrm{Mb}$ to $72.3 \mathrm{Mb}$ (see Fig. 1c). Out of these merged ROHs, 31 ranging in size from $0.4 \mathrm{Mb}$ to $5.8 \mathrm{Mb}$ were shared in more than half of the affected individuals (see Fig. 1c and Supplementary Table 5). The two ROHs most commonly shared between affected individuals were a $4.7 \mathrm{Mb}$ region on chromosome 3 (chr3: 48,187,679-52,913,780) and a 1.6 Mb region on chromosome 4 (chr4: 33,294,517-34,943,265). The chromosome 3 region spans 159 genes, including neuronal development genes SEMA3F and SEMA3B, and epilepsy-associated genes NPRL2, CACNA2D2, and CYB561D2 (see Supplementary Fig. 7a). The chromosome 4 region contains three long non-coding RNAs (see Supplementary Fig. 7b).

We examined the burden of damaging mutations within $\mathrm{ROHs}$ by comparing the rate of rare LoF and MD variants within and outside $\mathrm{ROHs}$ for all individuals. We found that the rate of rare homozygous LoF and MD variants within $\mathrm{ROH}$ s were significantly higher than the rest of the genome (see Fig. 2a). We then examined the distribution of rare homozygous variation across the genome, and found that ROHs, which on average spanned $9.65 \%$ of the genome in our cohort, harbor $29.5 \%$ of rare homozygous LoF/MD variants (see Fig. 2b). This percentage did not differ significantly for rare homozygous ND variants within $\mathrm{ROHs}(34.6 \%)$ (see Fig. 2b), indicating that ROHs do not necessarily carry more damaging variation, but are rather enriched for all rare homozygous variation. Using data from gnomAD, we assessed the constraint of genes carrying rare inherited homozygous LoF and MD variants, and found that such genes within ROHs showed higher constraint and intolerance to homozygous LoF mutations (average pRec score $=0.5497$, average pNull score $=0.2503, N=$ 15) compared to genes that carry the same category of variants but are outside ROHs (average pRec score $=0.3104$, average pNull score $=0.5404, N=14$ ) (see Fig. 2c). Constraint for genes carrying rare inherited homozygous ND variants did not change whether the genes were within or outside $\mathrm{ROH}$ s (see Fig. $2 \mathrm{~d}$ ).

To identify candidate ASD variants within ROHs, we excluded all $\mathrm{ROH}$ s that were found in any of the parents. We found $4 \mathrm{ROH}$ segments shared between two to four affected individuals, all smaller than $0.1 \mathrm{Mb}$ (see Supplementary Table 6). There were no coding variants shared between the affected individuals carrying these regions, however, there were 76 shared noncoding variants (see Supplementary Table 7). To better interpret the functional impact of these variants, we annotated them using three publicly available ChIP-seq and ATAC-seq datasets: (1) chromatin state segmentation from nine human cell lines ${ }^{33}$, (2) maps of the activating histone modification $\mathrm{H} 3 \mathrm{~K} 4 \mathrm{me} 3$ in human prefrontal cortex from 11 individuals ${ }^{34}$, and (3) predicted developmental brain enhancers from human fetal brain samples ${ }^{35}$. We identified an $\mathrm{ROH}$ on chromosome 3 (chr3: 18,335,384-18,359,238) carrying a shared variant that mapped to a predicted heterochromatic region. This $\mathrm{ROH}$ spans SATB1, which encodes an activitydependent transcription factor that regulates neuronal development $^{36,37}$, and LOC339862, a IncRNA gene found to be hypermethylated in senile plaques of postmortem human brains with Alzheimer's disease ${ }^{38}$. Another shared $\mathrm{ROH}$ on chromosome 4 (chr4: 3,389,923-3,476,537) harbored several intronic variants that mapped onto predicted enhancers and transcriptional elongation/ transition-related regions. The ROH spans RGS12 which encodes a G-protein signaling regulator and transcriptional repressor with enhanced expression in the brain (based on data from the Genotype-Tissue Expression Portal, GTEx), and DOK7, which encodes a protein involved in neuromuscular junction formation, and shows moderate expression in the brain and enhanced expression in heart and skeletal muscle (GTEx). Another interesting shared $\mathrm{ROH}$ on chromosome 22 harbored a variant in a transcriptional elongation/transition-related region in an intron of ZMAT5, an RNA splicing/processing gene that is highly expressed in the brain (GTEx) (see Supplementary Table 7).

\section{Identification of candidate ASD variants}

For candidate ASD variant discovery, we initially focused on rare exonic nonsynonymous or splice site variants in affected individuals that were either de novo or fit a recessive inheritance model. We identified an average of 80 de novo variants per proband (see Supplementary Table 8 ). This is slightly increased compared to previous reports of $\sim 60-70$ de novo variants per genome, however the average number of coding de novo variants was 1 per proband, similar to previous reports ${ }^{39,40}$. The lack of a public WGS dataset from a cohort of similar ancestry to our cohort, likely contributed to this moderate increase in the number of noncoding de novo variants. For coding de novo variants, on the other hand, we were able to filter against the GME, a whole exome sequencing dataset. In addition, we identified an average of 1251 inherited homozygous variants (6 coding) and 22 compound heterozygous variants in 10 genes per proband (see Supplementary Table 8). We also identified an average of 5 recessive $X$-linked variants in affected male offspring, none of which were coding (see Supplementary Table 8). We did not see a significant correlation between the number of de novo variants and maternal or paternal age at birth of an affected offspring which may be due to the small number of families for which parental ages were available (see Supplementary Fig. 8). In total, we identified 258 rare exonic nonsynonymous or splice site variants in 152 genes (see Supplementary Table 9). Out of these, 95 variants were in 42 OMIM-annotated disease genes associated with relevant 
a

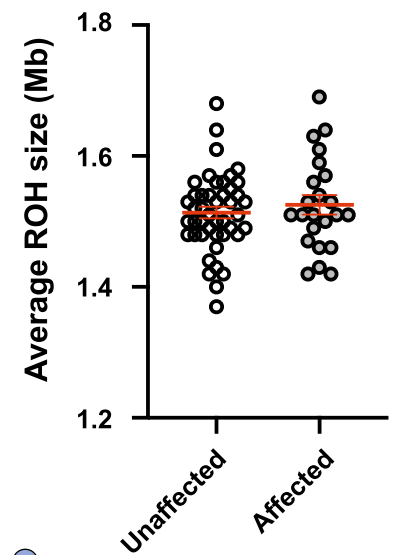

C

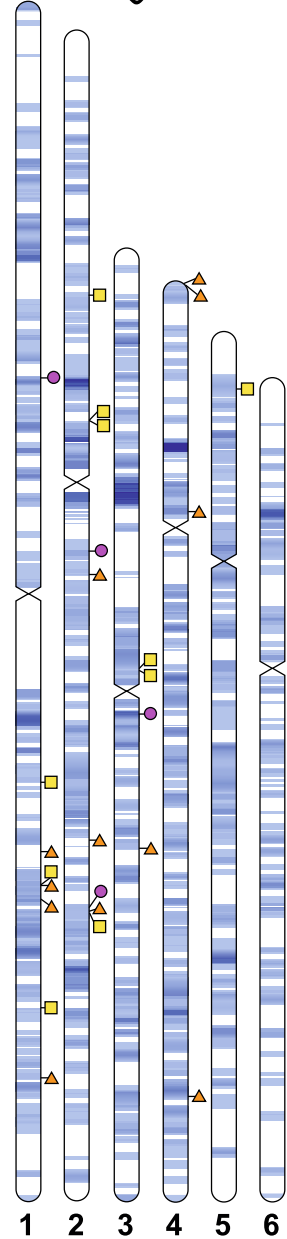

b

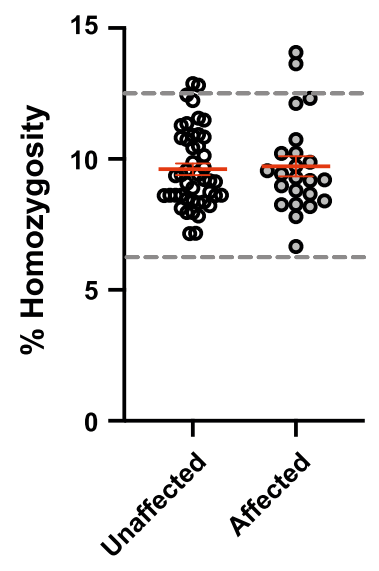

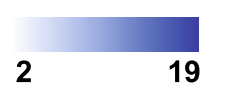
$\square$ enhancer
promoter
$\triangle$ splicing
$\triangle$ missense

Fig. 1 Runs of homozygosity in the consanguineous ASD cohort. There were no significant differences in average size of ROHs (a) or total percent of homozygosity across the genome (b) between affected and unaffected individuals (mean \pm SEM are shown in red). In $\mathbf{b}$ dotted lines denote the expected \% homozygosity for children of second- and third-degree relatives ( $12.5 \%$ and $6.25 \%$, respectively). Data were analyzed using unpaired $t$ test $(N=23$ affected, 45 unaffected; $P=0.4599(\mathbf{a}), P=0.7878(\mathbf{b}))$. c ROHs shared between unrelated probands, with rare inherited homozygous nonsynonymous variants and brain-specific regulatory element variants that map within them. The color scale indicates the number of probands sharing an $\mathrm{ROH}$.

phenotypes including intellectual disability, developmental delay, and epilepsy.

We cross-referenced the rare inherited homozygous variants within $\mathrm{ROH}$ s in affected individuals. We identified 63 rare homozygous coding variants (0-12 per genome) that were located within an $\mathrm{ROH}$ and were not present in homozygous form in the parents or an unaffected sibling (see Supplementary
Table 8). Out of these, 39 variants (0-9 per genome) were nonsynonymous (see Fig. 1c and Supplementary Tables 8 and 9).

The majority of inherited homozygous variants within ROHs were noncoding. Annotation of these noncoding variants with the aforementioned ChIP-seq and ATAC-seq datasets identified an average of $3(0-13)$ variants within predicted human brain promoters as well as an average of $2(0-12)$ variants within 
a

All

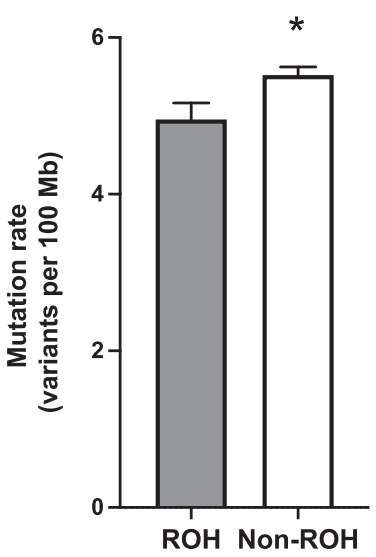

Heterozygous

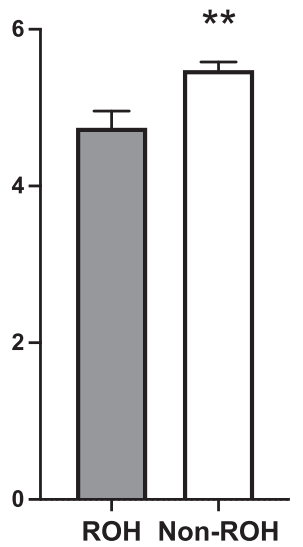

Homozygous

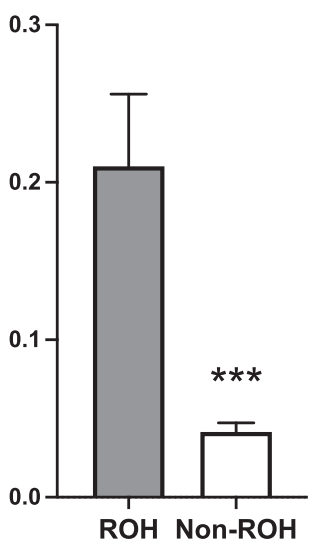

b

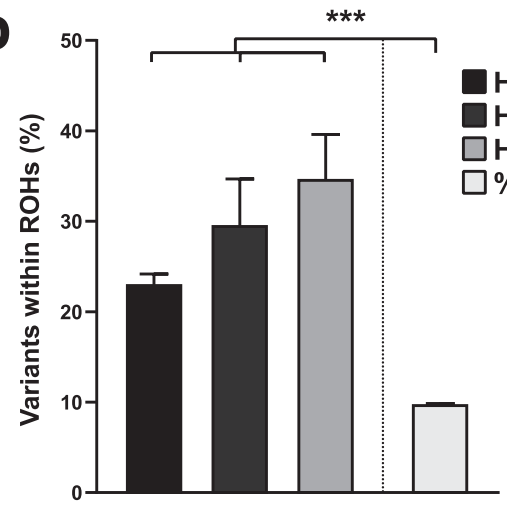

C

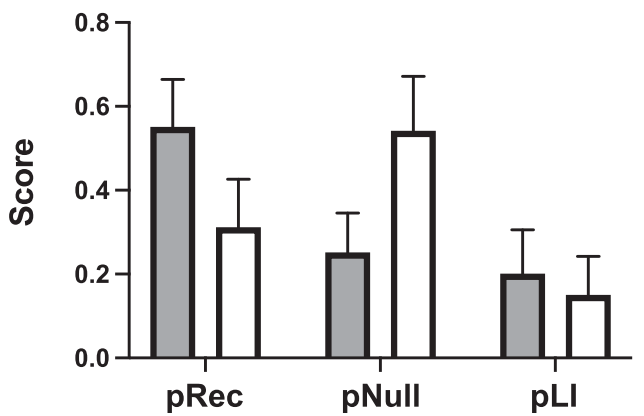

Homozygous

Homozygous LoF/MD

$\square$ Homozygous ND

$\%$ of Genome within ROHs 
showed weak to moderate associations with promoter, enhancer, and transcription elongation/transition regions based on the ENCODE dataset (see Supplementary Table 11). Two of these variants shared by two unrelated probands were located in the ASD-associated gene EXOC6.

We utilized multiple criteria to prioritize nonsynonymous coding and brain-specific noncoding variants as potentially causative for each affected individual. The prioritization or exclusion criteria for each variant are summarized in Supplementary Tables 9 and 10. Briefly, we considered (1) known association of the gene with ASD or other neurodevelopmental diseases, (2) gene expression in the brain from GTEx and Human Protein Atlas data $^{41}$, (3) protein damage prediction and evolutionary conservation for coding variants, and (4) validation of the predicted promoter/enhancer interactions in a fourth human brain dataset for noncoding variants ${ }^{42}$. We identified 46 candidate variants in 36 genes or human brain-specific regulatory elements in 20 affected individuals (see Tables 2 and 3).

Variants in known ASD or neurodevelopmental disease genes. Table 2 summarizes the potentially pathogenic variants in known ASD or neurodevelopmental disease genes for each affected individual. We identified 27 variants in 14 affected individuals ( 1-3 per individual), including coding variants in 12 genes, variants in 5 brain-specific regulatory regions ( 3 in promoters, 2 in enhancers), and 3 CNVs.

Five affected individuals had coding variants in syndromic ASD genes: JC-50-3 in KMT2C, JC-57-3 in ZNF292, JC-60-3 in SYNE1, MC04-3 in SPTBN1, and MC-17-3 in SCN1A. Proband MC-04-3 presented with ASD, speech abnormalities, and developmental delay, in line with phenotypes of patients with pathogenic SPTBN1 mutations (MIM \#619475) ${ }^{43,44}$. In addition to ASD, proband MC-173 presented with epilepsy, the characteristic phenotype in patients with SCN1A loss of function mutations. Five other probands (JC-353, JC-37-3, JC-41-3, JC-58-3, and MC-32-3) had coding variants in neurodevelopmental disease genes: RTTN (MIM \#614833) ${ }^{45}$, RPGRIP1L (MIM \#619113, \#611560, \#611561) 46-48, CDK10 (MIM \# $617694)^{49}$, AARS (MIM \#613287, \#616339) 50,51 , TENM3 (MIM $\# 615145)^{52}$, and DCHS1 (MIM \#601390) ${ }^{53-55}$. In addition to ASD, individual JC-35-3 presented with intellectual disability and lack of speech, both of which are phenotypes associated with recessive loss of function mutations in $R T T N^{45,56,57}$. RPGRIP1L mutations are known to cause Joubert (MIM \#213300) ${ }^{48}$, Meckel (MIM \#611561), and $\mathrm{COACH}$ (MIM \#216360) ${ }^{46}$ syndromes, characterized by intellectual disability and gross brain defects. TENM3 encodes a transmembrane protein involved in the regulation of neuronal development ${ }^{58}$. Recessive TENM3 mutations have been identified in syndromic microphthalmia with developmental delay and speech abnormalities, and some cases presenting with intellectual disability $^{52}$. CNVs and noncoding variants in this locus have been previously associated with $\mathrm{ASD}^{59-61}$. Recessive mutations in DCHS1 result in Van Maldergem syndrome (MIM \#601390) ${ }^{53,54}$. Patients with Van Maldergem syndrome have been reported to have ASD, lack of speech, and developmental delay ${ }^{55}$, all of which are phenotypes of proband MC-32-3. For proband MC-14-3, our CNV analysis identified one small deletion within 1q43 and one duplication spanning the 1q43-1q44 boundary. Microdeletions and microduplications within 1q43-q44 are associated with neurodevelopmental phenotypes including intellectual disability, developmental delay, and limited or no speech ${ }^{62,63}$ (MIM \#612337), all of which were present in the proband. Furthermore, both of these CNVs overlapped with SFARI-annotated ASD genes: the deletion is located within an intron of FMN2 and the duplication spans HNRNPU and ADSS2.

In addition to coding variants, we identified noncoding variants in three affected individuals that mapped to 5 loci predicted to be brain-specific regulatory regions for known ASD and neurodevelopmental disease genes (see Table 2).
These noncoding variants were within promotor or enhancer elements for the ASD genes AUTS2, CNTNAP4, and CTNND2, as well as neurodevelopmental disease genes ACTG1 (MIM \#614583) ${ }^{64}$ and SPTBN4 (MIM \#617519) ${ }^{65}$.

Variants in new candidate ASD genes. We identified 15 potentially pathogenic coding variants in 12 candidate ASD genes (see Table 3). Several of the candidate ASD genes that we identified encode proteins that function in neuronal development and connectivity (e.g., CHD5, GRB10). CHD5 is a chromatin remodeler that regulates neuronal differentiation and cortical development ${ }^{66}$, and its disruption in mice results in ASD-like behaviors ${ }^{67}$. A recent report identified heterozygous missense and LoF mutations in CHD5 in an autosomal dominant neurodevelopmental disorder of intellectual disability, developmental delay, language deficits, and epilepsy ${ }^{68}$. GRB10 is involved in neuronal development and social dominance behavior in mice, and has a tissue-specific and imprinted expression pattern where the paternal allele is exclusively expressed in neurons ${ }^{69}$. GRB10 is also a known interactor of GIGYF1, encoded by a high-confidence ASD risk gene $^{70}$. Mutations in the cation-encoding gene TRPV4 are associated with motor neuropathy and sensory abnormalities ${ }^{71}$.

We identified 6 noncoding (3 promoter, 3 enhancer) candidate ASD variants that are located within human brain-specific regulatory regions (see Table 3 ). These variants are located in regulatory regions for neuropsychiatric and neurodegenerative disease genes, including TSNARE $1^{72}$ and $A D C Y 9^{73}$. Other genes predicted to be affected by a noncoding variant include SMG7, involved in nonsense-mediated mRNA decay regulation, a pathway that has been previously implicated in ASD and related disorders $^{74,75}$. Differential methylation of the SMG7 and SMG7-AS1 promoters was associated with SMG7 overexpression in an ASD patient ${ }^{76}$. Interestingly, proband MC-14-3 carries a deleterious coding variant within SMG7 as well as the enhancer variant. Another interesting variant was identified in affected siblings MC24-3 and MC-24-4, in an enhancer region that interacts with the promoters of TSNARE1, a gene associated with schizophrenia, and $A D G R B 1$, a critical regulator of spine and synapse development ${ }^{77}$. Conserved transcription factor binding site variants in ADGRB1 have also been previously identified in ASD patients ${ }^{8}$.

\section{DISCUSSION}

We performed WGS in a consanguineous cohort consisting of 68 individuals in 22 families, with at least one child in each family diagnosed with ASD. We used homozygosity mapping to identify runs of homozygosity and analyzed rare alleles in these segments. The largest autosomal $\mathrm{ROH}$ identified was a $7.6 \mathrm{Mb}$ segment found in an affected individual. The average fraction of the genome under $\mathrm{ROHs}$ was $9.65 \%$, larger than the expected percentage of shared homozygosity for children of first cousins $(6.25 \%)^{78}$. The size of the larger blocks of homozygosity we observed are consistent with observations in other consanguineous cohorts and are indicative of previous generations of consanguinity ${ }^{78}$. Analysis of shared $\mathrm{ROHs}$ between affected individuals in our cohort showed no common genomic region harboring potentially causative variants. We also did not identify any deleterious coding, human brain-specific noncoding, or copy number variation within $\mathrm{ROH}$ s that were shared across unrelated probands. This once again highlights the genetic heterogeneity of ASD, and indicates that for ASD, analysis of $\mathrm{ROHs}$ is more informative when done on a within-family basis, to help identify potentially causative variants in each affected individual. Burden analysis showed that rare inherited homozygous LoF and MD variants are enriched in $\mathrm{ROHs}$, and that the genes harboring these mutations are less likely to tolerate such mutations (see Fig. 2). This supports what is known about consanguinity in that it increases the risk of recessive disease in the offspring, and demonstrates that 


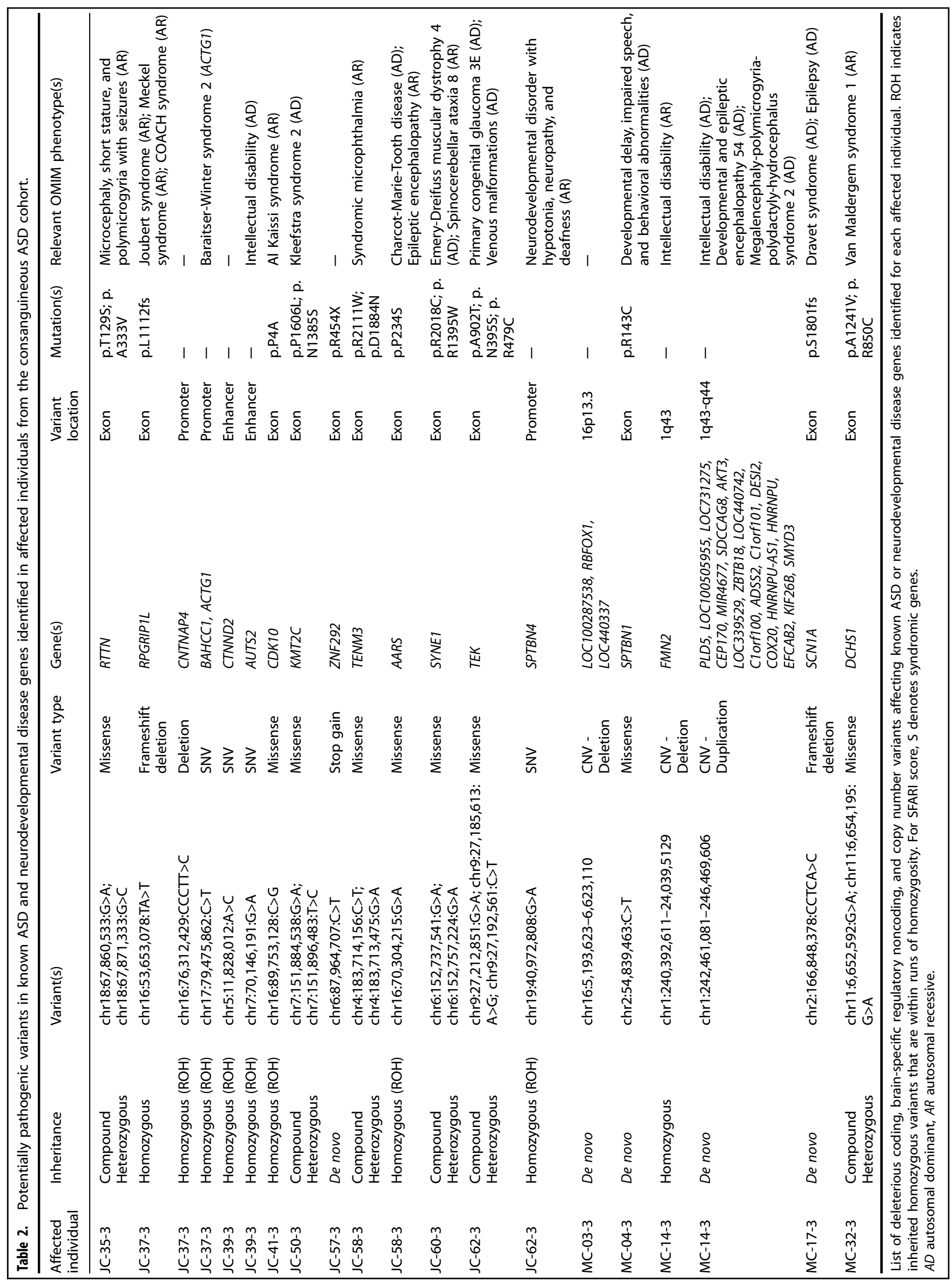




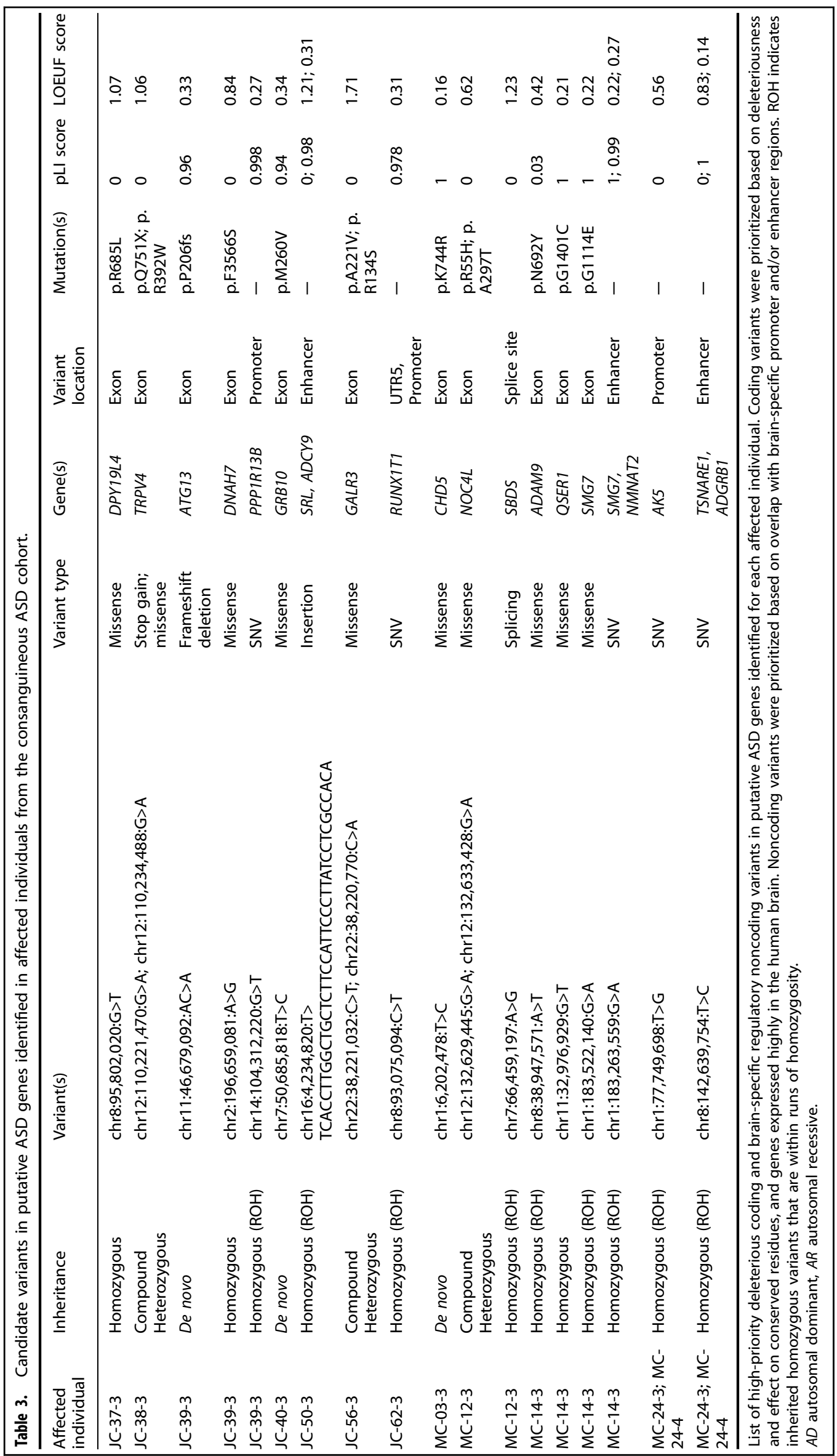


homozygosity mapping is an effective method to identify genomic regions that likely harbor recessive disease-causing mutations. We analyzed ROHs absent from parents and unaffected siblings and shared between unrelated probands, and we identified rare homozygous variants within these regions that were also shared between the probands. We identified 4 genomic regions each harboring at least one brain-expressed gene, as well as rare homozygous variants within these genes that were shared between affected individuals carrying each $\mathrm{ROH}$. While homozygous events are enriched in consanguineous cohorts, compound heterozygous and de novo events are also expected. Our analysis identified 34 potentially pathogenic rare coding variants in 24 genes (see Tables 2 and 3). Eighteen of the 24 candidate genes carried biallelic events, and for 5 of these genes, these were located within an $\mathrm{ROH}$. In addition, we identified 37 rare homozygous $\mathrm{ROH}$ variants that were located within brainspecific regulatory elements, 5 of which were located within or near a known ASD gene (see Supplementary Table 10). We also identified CNVs that overlap with known ASD CNV regions in 10 affected individuals (see Supplementary Table 3).

Tables 2 and 3 summarize the variants we identified in known ASD and neurodevelopmental disease genes and in new candidate ASD genes in each affected individual, respectively. In each of 12 affected individuals, we identified a single exonic variant (or 2 variants in case of compound heterozygosity) segregating with phenotype and meeting our criteria for deleteriousness, making these variants putatively pathogenic and the respective genes potentially causative. Five of these genes are known ASD genes (KMT2C, SCN1A, SPTBN1, SYNE1, ZNF292), 3 underlie neurodevelopmental disease but have not been previously implicated in ASD (CDK10, DCHS1, RTTN), and 4 are new candidate ASD genes (CHD5, GALR3, GRB10, TRPV4). For three probands, no candidate ASD variants were identified, and for the remaining 8 affected individuals, we identified $\sim 2-6$ candidate ASD variants, including exonic de novo or biallelic variants and homozygous variants in human brain-specific regulatory regions. In these cases, with multiple rare potentially pathogenic variants including those in $\mathrm{ROHs}$, further functional investigation is needed to determine the potential causality of the identified variants.

Although consanguineous cohorts are rich for biallelic events, de novo variation is still present and is expected to contribute to disease burden. Five probands each carried 1 de novo candidate variant and no candidate biallelic events. MC-04-3 carries a de novo missense variant in the ASD gene SPTBN $1{ }^{43,44}$. Proband MC17-3 carries a de novo frameshift deletion in $S C N 1 A$, in line with his epilepsy phenotype, and proband JC-57-3 carries a de novo nonsense variant in the syndromic ASD gene ZNF292. This mutation (p.R454X) has previously been reported in a patient with mild developmental and speech delays but not with ASD $^{79}$. For each of JC-40-3 and MC-03-3, single candidate genes with no prior implication in any neurodevelopmental disease were identified, suggesting GRB10 and CHD5 as putative ASD genes. Two additional putative ASD genes were identified in probands carrying compound heterozygous variants: JC-38-3 in TRPV4 and JC-56-3 in GALR3.

By utilizing WGS and homozygosity mapping, we were able to identify candidate biallelic variants within human brain-specific regulatory regions for known ASD and neurodevelopmental disease genes as well as new candidate ASD genes. In family MC-24, the two affected siblings shared a candidate ASD variant in an enhancer element linked to TSNARE1 and ADGRB1, two genes with known neuronal functions ${ }^{77,80,81}$, as well as associations to $\mathrm{ASD}^{8}$, schizophrenia ${ }^{72}$, and Parkinson's disease ${ }^{82}$. Another affected individual, JC-39-3, carried homozygous variants in brain-specific enhancers for 2 known ASD genes, AUTS2 and CTNND2. Identification of additional regulatory element variants and further characterization of their functional impact will contribute to our understanding of ASD etiology and the landscape of ASD genetics.

In total, our analysis revealed potentially etiological ASD variants in 14 out of 23 affected individuals. We provide further evidence of the contribution of biallelic events to ASD and of the importance of analyzing genomic data from consanguineous cohorts to identify rare recessive coding variants, as well as evaluating the potential contribution of noncoding variants. This approach is an effective means of discovering genes underlying ASD, promoting further investigation and understanding of the biological underpinnings of disease.

\section{METHODS}

\section{Subjects and specimens}

All human studies were reviewed and approved by the institutional review board (IRB) of the University of Texas Southwestern Medical Center (UTSW), the research committee at the University of Jordan School of Medicine, the ethics committee of the Jordan University Hospital, and the IRB of the Jordan University of Science and Technology. Families were recruited either from Jordan or from the Dallas Fort Worth area and written informed consent was obtained from all study participants. Inclusion criteria included a diagnosis of autism spectrum disorder (ASD) by a neurologist, child psychiatrist, or psychologist. Patients with genetically defined syndromes, specifically Fragile $X$ syndrome, Angelman syndrome, Rett syndrome, or Tuberous sclerosis complex, were excluded from study participation. All patients enrolled in the study received a diagnosis of ASD from their referring clinicians who performed physical and behavioral assessments and administered the standard autism diagnostic measures (ADOS, ADI-R, and DSM-V). Blood samples were collected from all available family members by peripheral venipuncture and genomic DNA was isolated from circulating leukocytes using AutoPure (Qiagen, Hilden, Germany) according to the manufacturer's instructions.

\section{Whole genome sequencing and data processing}

Sequencing was performed in collaboration with the Autism Speaks MSSNG Consortium ${ }^{83}$ and was carried out at The Centre for Applied Genomics at The Hospital for Sick Children (Toronto, Canada) on the Illumina HiSeq X platform as previously described ${ }^{83-85}$. Briefly, DNA quality and quantity were assessed using a Qubit High Sensitivity Assay. Between $100 \mathrm{ng}$ and $1 \mu \mathrm{g}$ of DNA was used for genomic library preparation using the Illumina TruSeq Nano DNA Library Prep Kit according to the manufacturer's protocol and libraries were paired-end sequenced (150 bp read lengths) ${ }^{86}$.

The genomes were processed as previously described ${ }^{83}$ following the best practices recommended by the Broad Institute ${ }^{87}$. Reads were aligned to the human reference genome version GRCh37/hg19 using the BurrowsWheeler Aligner (BWA, version 0.7.10). Duplicate reads were removed using Picard (version 1.117). Local realignment and quality recalibration were performed using the Genome Analysis Toolkit (GATK; version 3.3). Variants (single nucleotide variants (SNVs) and insertions or deletions (indels)) were detected using GATK with HaplotypeCaller. Quality control checks for (i) duplicate samples, (ii) samples per platform, (iii) genome call rate, (iv) missingness rate, (v) singleton rate, (vi) heterozygosity rate, (vii) homozygosity rate, (viii) Ti/Tv ratio, (ix) inbreeding coefficient, and ( $x$ ) sex inference were performed as previously described ${ }^{83}$. Variant call format (VCF) files for SNVs and indels were annotated with ANNOVAR using allele frequencies from the 1000 Genomes project $(2015 ; 1000 \mathrm{G})^{26}$, the Genome Aggregation Database (gnomAD) ${ }^{27}$, and the Greater Middle East Variome Project $(\mathrm{GME})^{28}$. Annotated VCF files were uploaded into a SQL database for working storage and analysis. Genome data was stored and analyses were performed on the Texas Advanced Computing Center (TACC) high-performance computing servers, a resource of the University of Texas (Austin, TX)

\section{Variant filtration}

Variants were quality filtered in SQL using the PASS filter, a genotype quality (GQ) score of $\geq 99$, and allelic read depth of $\geq 10$. Rare variants were defined as those with minor allele frequencies (MAF) $<1 \%$ in $1000 \mathrm{G}$, gnomAD, and GME. Ultra-rare variants were identified by filtering for $\mathrm{MAF}=0$ in $1000 \mathrm{G}$, gnomAD, and GME. Private variants were defined as ultra-rare variants that occurred only in a single individual in our cohort. 
De novo variants were defined as any variant not present in the genome of either the father, the mother, or the sibling of a proband when available. To minimize potential false positive de novo calls, we applied additional filtering steps, requiring that de novo variants have the following criteria: (i) the ratio of sequence reads supporting the alternative call to total calls is between $0.3-0.7$ for all variants in female probands and all autosomal and pseudoautosomal variants in male probands, and $\geq 0.7$ for variants on the $X$ and $Y$ chromosomes in male probands, (ii) $Q D \geq 4$ and ReadPosRankSum $\geq-2.5^{39}$, (iii) the variant does not overlap with any rare variant in any other individual in our cohort, (iv) variant $\mathrm{MAF}<0.1 \%$ in 1000G, gnomAD, and GME, (v) variant size of $<50$ bp for indels, (vi) the variant does not fall within known segmental duplications or simple repeat regions. To identify compound heterozygous variants in affected individuals, we selected rare coding (exonic or splicing) heterozygous events that were present in heterozygous form in one parent but not the other, then filtered for inherited variants within the same gene. Homozygous inherited variants were required to be present in heterozygous form in both the father and the mother, excluding variants that are homozygous in one of the parents on the assumption of full penetrance. X-linked variants were present in a male offspring and heterozygous in the mother. We also excluded pseudoautosomal variants that were heterozygous in the male offspring, and X-linked variants present in the unaffected father.

\section{Noncoding variant annotation}

Custom SQL and Python scripts were used to annotate noncoding variants using three datasets: (1) chromatin state segmentation from nine human cell lines ${ }^{33}$, (2) maps of histone H3K4me3 mark in human prefrontal cortex (PFC) from 11 individuals ${ }^{34}$, and (3) predicted developmental brain enhancers from fetal brain samples ${ }^{35}$. The columns in supplementary tables derived from each dataset were denoted as ENCODE, uMass, and CBA, respectively. Additional details are presented in Supplementary Table 12. Variants that were found within a peak in the uMass dataset were marked as "predicted human brain promoter" variants. Predicted human brain promoter variants that were absent from regions with "1_Active_Promoter" prediction in any one of the 9 non-neuronal cell lines in the ENCODE dataset were marked as "predicted human brain-specific promoter" variants. Variants that were found within a predicted regulatory element (pRE) region in the CBA dataset were marked as "predicted human brain enhancer" variants. Predicted human brain enhancer variants that were absent from regions with "4_Strong_Enhancer" or "5_Strong_Enhancer" prediction in any of the 9 non-neuronal cell lines in the ENCODE dataset were marked as "predicted human brain-specific enhancer" variants. To verify regulatory element prediction, brain-specific enhancer and promoter variants were visualized using a UCSC genome browser track of brain cell-type specific proximity ligation-assisted ChIP-seq (PLAC-seq) data from Nott et al. ${ }^{42}$. PLAC-seq identifies long-range chromatin interactions at promoters and enhancers. We marked variants as linked to a certain gene if the enhancer region where the variant is located (based on the ChIP-seq and ATAC-seq data from the aforementioned UCSC genome browser tracks) was linked to the promoter of the target gene in the PLAC-seq data.

\section{Variant prioritization}

For individual JC-35-3, compound heterozygous and homozygous inherited variants that were present in unaffected sibling JC-35-5 were excluded from analysis. For MC-24-3 and MC-24-4, compound heterozygous and homozygous inherited variants that were not shared by the affected siblings were excluded. De novo, compound heterozygous, inherited homozygous, and X-linked variants that are rare were considered to be potentially pathogenic if they met the following criteria: exonic or splice site, with an effect on the protein resulting in either a frameshift indel, a stopgain or stoploss, or nonsynonymous. We also kept variants with "unknown effect" to retain splice site variants in our analysis. For missense variants, we considered those that met at least two of the following criteria, with specific score cutoffs based on the cited published literature: SIFT score $<0.05^{88}$, PolyPhen-2 HumVar score $>0.15^{89}$, PROVEAN score $<-2.5^{90}$, and MutationAssessor score $>2.26^{91}$. PolyPhen-2 HumVar was chosen over PolyPhen-2 HumDiv because the former is more appropriate for Mendelian variants with drastic effect as we expect for ASD, while the latter is appropriate for common variants of smaller effect size. To assess evolutionary conservation of residues with missense variants, we considered CADD ${ }^{92}$, phastCons, phyloP ${ }^{93}$, and GERP $++{ }^{94}$ scores. Gene constraint was assessed using gnomAD pLI and Z scores ${ }^{27}$.
We also inspected conservation across species by identifying gene homology groups using NCBI HomoloGene, and visualizing in Geneious Prime using Clustal Omega alignments ${ }^{95}$. The filtered variants were compared to the list of genes implicated in ASD from the Simons Foundation Autism Research Initiative (SFARI) Gene 2018 database $^{6}$ (using the latest version updated in February 2020) and a list of established neurodevelopmental disease genes ${ }^{7}$. Variants were also screened for any phenotypic association in the Online Mendelian Inheritance in Man (OMIM) database. As an additional prioritization step, we deprioritized coding variants with $\mathrm{MAF} \geq 1 \%$ and noncoding variants with a $\mathrm{MAF}>0.1 \%$, or incidences of any homozygous alleles, in each subpopulation of $1000 \mathrm{G}$ and gnomAD. We especially focused on subpopulations relevant to the ancestry of each family in our cohort (i.e. SAS for MC-03, MC-17, MC-21, MC-32; EUR and EAS for MC-12; EUR and ASJ for MC-16; EUR for MC-04 and MC-24; and GME Syrian desert subpopulation for all Jordanian families).

Each potentially pathogenic coding or brain-specific noncoding variant was considered as high, medium, or low priority based on the criteria above. Priority rankings and the specific ranking criteria are included in Supplementary Tables 9 and 10. We selected high priority variants for each affected individual as the potentially pathogenic candidate ASD variants (Tables 2 and 3 ). If an affected individual did not carry any variants that were high priority, we selected medium priority variants.

\section{Copy number variant (CNV) analysis}

We used CNVkit ${ }^{29}$ to detect CNVs based on read depth in affected samples relative to the average read depth in unaffected samples in the same family as controls, following general protocols ${ }^{96}$. We used GISTIC $2.0^{30}$ on segmented files generated from CNVkit ${ }^{29}$ to further evaluate the significance of the amplified and deleted segments between the affected and unaffected samples. The criteria included a threshold for copy number amplification and deletion of 0.1 , confidence level of $99 \%$, and FDR of 0.05 . Proband CNVs that overlap with SFARI-annotated ASD CNVs were identified using $\mathrm{R}$ Bioconductor package regione $\mathrm{R}^{97}$. Significance of the overlaps was tested by performing an overlap permutation test, also using regione $R^{97}$.

\section{Burden analysis}

Nondisrupting (ND) variants were defined as exonic synonymous SNVs or exonic non-frameshift indels. Missense damaging (MD) variants were defined as exonic nonsynonymous SNVs that met at least two of the following criteria: SIFT score $<0.05$, PolyPhen-2 HumVar score $>0.15$, PROVEAN score $<-2.5$, and MutationAssessor score $>2.26$. Loss of function (LoF) variants were defined as splice site variants, and exonic variants predicted to result in a stopgain, stoploss, or frameshift indel. For the total burden analysis, the numbers of rare variants in each category (ND, MD, LoF) were compared between affected and unaffected individuals. For the analysis of burden within $\mathrm{ROHs}$, mutation rates for ND and for LoF/MD variants were calculated by dividing the number of variants by the total length of $\mathrm{ROHs}$ for each individual. For the analysis of gene constraint, average pRec, pNull, and pLI scores from gnomAD were compared for genes carrying rare inherited homozygous ND or LoF/MD variants that are within or outside of a $\mathrm{ROH}$ in the affected individual who carries the variant. Briefly, pRec, pNull, and pLI describe the probability that a particular gene is recessive, unconstrained, or LoF-intolerant (likely haploinsufficient), respectively ${ }^{27}$. Genes with higher $\mathrm{pRec}$ and $\mathrm{pLI}$ scores are more likely to be intolerant to homozygous and heterozygous LoF variants, respectively. Genes with higher pNull scores are more likely to be completely tolerant of LoF variation.

\section{Assessment of runs of homozygosity}

PLINK version $1.90 \mathrm{~b} 6.7^{98,99}$ was used for all analyses. VCF files were converted into PLINK format using vcftools version 0.1.13. The cohort was assessed for relatedness using PLINK-genome. Variants were filtered for Hardy-Weinberg equilibrium $(P<0.001), \mathrm{MAF}>5 \%$, and maximum missing genotype rate of $25 \%$. Runs of homozygosity (ROHs) were identified in PLINK using a sliding window analysis with a 100 base pair window size, allowing for 30 heterozygous variants and 30 missing genotypes per window in accordance with previously described methods ${ }^{18}$. The resulting segments were then assessed using the percent homozygous (PHOM) output from PLINK using thresholds of $50 \%, 70 \%, 75 \%$, and $80 \%$. The homozygosity threshold of $75 \%$ yielded genome-wide homozygosity in 
$\mathrm{ROH}$ s that were in accordance with expectations of homozygosity from relatedness determined through PLINK-genome and this threshold was used to filter $\mathrm{ROH}$ s for further analysis. Per-genome $\mathrm{ROH}$ metrics were calculated using autosomal ROHs only. Percentage of genome within $\mathrm{ROH}$ s was estimated as the ratio of total $\mathrm{ROH}$ length to total bases sequenced at $1 \times$. Custom Python scripts were used to identify ROHs that were present in affected individuals and absent from their respective parents, and to identify the boundaries and the counts for ROHs that were shared between affected individuals. $\mathrm{R}$ package Rldeogram was used to visualize shared $\mathrm{ROH}^{100}$.

\section{Variant validation}

Candidate variants selected for validation were either in runs of homozygosity, or were de novo heterozygous or compound heterozygous variants in genes from the SFARI Gene 2018 database or from a list of neurodevelopmental disease genes ${ }^{7}$. A total of 61 candidate variants were assessed by targeted Sanger sequencing and 60 were validated (Supplementary Table 13). Genomic sequence surrounding the variant was downloaded from the University of California, Santa Cruz (UCSC) genome browser version GRCh37/hg19. PCR primers were designed to isolate and amplify the region surrounding a variant using the NCBI Primer-BLAST. Sequencing primers were designed using Primer3 version 0.4.0. PCR was carried out using standard protocols. Sequencing was performed at Genewiz (South Plainfield, NJ) or at the Eugene McDermott Center for Human Growth and Development Center Sequencing Core Facility at UTSW (Dallas, TX). The list of validated variants and sequences of the primers used can be found in Supplementary Table 13.

\section{Principal component analysis}

Principal component analysis (PCA) was carried out in PLINK version 1.90b6. $7^{98,99}$ using Phase 3 1000G data. PCA input files from our samples were created from VCF files using vcftools version 1.13 and were pruned to remove variants with $\mathrm{MAF}<5 \%$, missing genotype rate greater than $5 \%$, and pruned for linkage disequilibrium (LD) with an $r^{2}$ threshold of 0.2 using PLINK -indep-pairwise 505 0.2. Triallelic and palindromic variants were also removed. The set of variants that remained was extracted from the $1000 \mathrm{G}$ dataset and these were merged with our cohort dataset. PCA was run in PLINK using the -pca flag and the first two principal components were plotted in R.

\section{Web resources}

1000G: https://www.internationalgenome.org/data; Allen Brain Atlas: http://www.brain-map.org; gnomAD Browser: http://gnomad. broadinstitute.org; GME: http://igm.ucsd.edu/gme; GTEx Portal: https:// www.gtexportal.org/home; NCBI Primer-BLAST: https://www.ncbi.nlm.nih. gov/tools/primer-blast/; OMIM: http://www.omim.org; PLINK: http://pngu. mgh.harvard.edu/purcell/plink/; Primer3: http://bioinfo.ut.ee/primer3-0.4.0/ ; UCSC Genome Browser: http://genome.ucsc.edu.

\section{Reporting summary}

Further information on research design is available in the Nature Research Reporting Summary linked to this article.

\section{DATA AVAILABILITY}

Sequence data can be accessed through the Autism Speaks MSSNG database (for access, see https://research.mss.ng/). MSSNG is a well-established whole genome sequence resource utilized by approved investigators worldwide. Sequence data have also been deposited at the European Genome-phenome Archive (EGA), which is hosted by the EBI and the CRG, under accession number EGAS00001005938.

\section{CODE AVAILABILITY}

Codes used in the MSSNG database have been published ${ }^{83}$ and can be accessed here: https://www.ncbi.nlm.nih.gov/pmc/articles/PMC5501701/. Others are available upon request.

Received: 13 July 2021; Accepted: 21 January 2022; Published online: 21 February 2022

\section{REFERENCES}

1. Maenner, M. J. et al. Prevalence and characteristics of autism spectrum disorder among children aged 8 years-autism and developmental disabilities monitoring network, 11 sites, United States, 2018. Morbidity Mortal. Wkly. Report. Surveill. Summaries 70, 1-16 (2021).

2. Fernandez, B. A. \& Scherer, S. W. Syndromic autism spectrum disorders: moving from a clinically defined to a molecularly defined approach. Dialogues Clin. Neurosci. 19, 353-371 (2017).

3. Colvert, E. et al. Heritability of autism spectrum disorder in a UK populationbased twin sample. JAMA Psychiatry 72, 415-423 (2015).

4. Sandin, S. et al. The heritability of autism spectrum disorder. JAMA 318, 1182-1184 (2017)

5. Tick, B., Bolton, P., Happe, F., Rutter, M. \& Rijsdijk, F. Heritability of autism spectrum disorders: a meta-analysis of twin studies. J. Child Psychol. Psychiatry 57, 585-595 (2016).

6. Basu, S. N., Kollu, R. \& Banerjee-Basu, S. AutDB: a gene reference resource for autism research. Nucleic Acids Res. 37, D832-D836 (2009).

7. Betancur, C. Etiological heterogeneity in autism spectrum disorders: more than 100 genetic and genomic disorders and still counting. Brain Res. 1380, 42-77 (2011).

8. Turner, T. N. et al. Genomic patterns of de novo mutation in simplex autism. Cell 171, 710-722e712 (2017).

9. Sanders, S. J. et al. De novo mutations revealed by whole-exome sequencing are strongly associated with autism. Nature 485, 237-241 (2012).

10. Dias, C. M. \& Walsh, C. A. Recent advances in understanding the genetic architecture of autism. Annu Rev. Genomics Hum. Genet. 21, 289-304 (2020).

11. Autism Spectrum Disorders Working Group of The Psychiatric Genomics Consortium. Meta-analysis of GWAS of over 16,000 individuals with autism spectrum disorder highlights a novel locus at 10q24.32 and a significant overlap with schizophrenia. Mol. Autism 8, 21 (2017).

12. $\mathrm{Ma}, \mathrm{D}$. et al. A genome-wide association study of autism reveals a common novel risk locus at 5p14.1. Ann. Hum. Genet. 73, 263-273 (2009).

13. Wang, $K$. et al. Common genetic variants on $5 p 14.1$ associate with autism spectrum disorders. Nature 459, 528-533 (2009).

14. Grove, J. et al. Identification of common genetic risk variants for autism spectrum disorder. Nat. Genet. 51, 431-444 (2019).

15. Satterstrom, F. K. et al. Large-scale exome sequencing study implicates both developmental and functional changes in the neurobiology of autism. Cell 180, 568-584 e523 (2020).

16. Tammimies, K. et al. Molecular diagnostic yield of chromosomal microarray analysis and whole-exome sequencing in children with autism spectrum disorder. JAMA 314, 895-903 (2015).

17. Doan, R. N. et al. Recessive gene disruptions in autism spectrum disorder. Nat. Genet. 51, 1092-1098 (2019).

18. Chahrour, M. H. et al. Whole-exome sequencing and homozygosity analysis implicate depolarization-regulated neuronal genes in autism. PLoS Genet. 8 e1002635 (2012).

19. Nalls, M. A. et al. Extended tracts of homozygosity identify novel candidate genes associated with late-onset Alzheimer's disease. Neurogenetics 10, 183-190 (2009).

20. Schuurs-Hoeijmakers, J. H. et al. Homozygosity mapping in outbred families with mental retardation. Eur. J. Hum. Genet. 19, 597-601 (2011).

21. Lencz, T. et al. Runs of homozygosity reveal highly penetrant recessive loci in schizophrenia. Proc. Natl Acad. Sci. USA 104, 19942-19947 (2007).

22. Wakeling, M. N. et al. Homozygosity mapping provides supporting evidence of pathogenicity in recessive Mendelian disease. Genet. Med. 21, 982-986 (2019).

23. Morrow, E. M. et al. Identifying autism loci and genes by tracing recent shared ancestry. Science 321, 218-223 (2008).

24. Casey, J. P. et al. A novel approach of homozygous haplotype sharing identifies candidate genes in autism spectrum disorder. Hum. Genet. 131, 565-579 (2012).

25. Novarino, G. et al. Mutations in BCKD-kinase lead to a potentially treatable form of autism with epilepsy. Science 338, 394-397 (2012).

26. The 1000 Genomes Project Consortium. et al. A global reference for human genetic variation. Nature 526, 68-74 (2015).

27. Karczewski, K. J. et al. The mutational constraint spectrum quantified from variation in 141,456 humans. Nature 581, 434-443 (2020).

28. Scott, E. M. et al. Characterization of Greater Middle Eastern genetic variation for enhanced disease gene discovery. Nat. Genet. 48, 1071-1076 (2016).

29. Talevich, E., Shain, A. H., Botton, T. \& Bastian, B. C. CNVkit: Genome-wide copy number detection and visualization from targeted DNA sequencing. PLoS Comput. Biol. 12, e1004873 (2016).

30. Mermel, C. H. et al. GISTIC2.0 facilitates sensitive and confident localization of the targets of focal somatic copy-number alteration in human cancers. Genome Biol. 12, R41 (2011). 
31. Lander, E. S. \& Botstein, D. Homozygosity mapping: a way to map human recessive traits with the DNA of inbred children. Science 236, 1567-1570 (1987).

32. Alkuraya, F. S. Discovery of rare homozygous mutations from studies of consanguineous pedigrees. Curr. Protoc. Hum. Genet Chapter 6, Unit6 12 (2012).

33. Ernst, J. et al. Mapping and analysis of chromatin state dynamics in nine human cell types. Nature 473, 43-49 (2011)

34. Cheung, I. et al. Developmental regulation and individual differences of neuronal H3K4me3 epigenomes in the prefrontal cortex. Proc. Natl Acad. Sci. USA 107, 8824-8829 (2010).

35. Markenscoff-Papadimitriou, E. et al. A chromatin accessibility atlas of the developing human telencephalon. Cell 182, 754-769 e718 (2020).

36. Close, J. et al. Satb1 is an activity-modulated transcription factor required for the terminal differentiation and connectivity of medial ganglionic eminencederived cortical interneurons. J. Neurosci. 32, 17690-17705 (2012).

37. Denaxa, M. et al. Maturation-promoting activity of SATB1 in MGE-derived cortical interneurons. Cell Rep. 2, 1351-1362 (2012).

38. Zhao, J. et al. A genome-wide profiling of brain DNA hydroxymethylation in Alzheimer's disease. Alzheimers Dement. 13, 674-688 (2017).

39. An, J. Y. et al. Genome-wide de novo risk score implicates promoter variation in autism spectrum disorder. Science 362, eaat6576 (2018).

40. Ruzzo, E. K. et al. Inherited and de novo genetic risk for autism impacts shared networks. Cell 178, 850-866 e826 (2019).

41. Uhlen, M. et al. Proteomics. Tissue-based map of the human proteome. Science 347, 1260419 (2015).

42. Nott, A. et al. Brain cell type-specific enhancer-promoter interactome maps and disease-risk association. Science 366, 1134-1139 (2019)

43. Cousin, M. A. et al. Pathogenic SPTBN1 variants cause an autosomal dominant neurodevelopmental syndrome. Nat. Genet. 53, 1006-1021 (2021).

44. Rosenfeld, J. A. et al. Heterozygous variants in SPTBN1 cause intellectual disability and autism. Am. J. Med. Genet. Part A 185, 2037-2045 (2021).

45. Kheradmand Kia, S. et al. RTTN mutations link primary cilia function to organization of the human cerebral cortex. Am. J. Hum. Genet. 91, 533-540 (2012).

46. Doherty, D. et al. Mutations in 3 genes (MKS3, CC2D2A and RPGRIP1L) cause $\mathrm{COACH}$ syndrome (Joubert syndrome with congenital hepatic fibrosis). J. Med. Genet. 47, 8-21 (2010).

47. Brancati, F. et al. RPGRIP1L mutations are mainly associated with the cerebellorenal phenotype of Joubert syndrome-related disorders. Clin. Genet. 74, 164-170 (2008).

48. Delous, M. et al. The ciliary gene RPGRIP1L is mutated in cerebello-oculo-renal syndrome (Joubert syndrome type B) and Meckel syndrome. Nat. Genet. 39, 875-881 (2007).

49. Windpassinger, $C$. et al. CDK10 Mutations in humans and mice cause severe growth retardation, spine malformations, and developmental delays. Am. J. Hum. Genet. 101, 391-403 (2017)

50. Latour, P. et al. A major determinant for binding and aminoacylation of tRNA (Ala) in cytoplasmic alanyl-tRNA synthetase is mutated in dominant axonal Charcot-Marie-tooth disease. Am. J. Hum. Genet. 86, 77-82 (2010).

51. Simons, C. et al. Loss-of-function alanyl-tRNA synthetase mutations cause an autosomal-recessive early-onset epileptic encephalopathy with persistent myelination defect. Am. J. Hum. Genet. 96, 675-681 (2015).

52. Singh, B., Srivastava, P. \& Phadke, S. R. Sequence variations in TENM3 gene causing eye anomalies with intellectual disability: Expanding the phenotypic spectrum. Eur. J. Med. Genet. 62, 61-64 (2019)

53. Cappello, S. et al. Mutations in genes encoding the cadherin receptor-ligand pair DCHS1 and FAT4 disrupt cerebral cortical development. Nat. Genet. 45, 1300-1308 (2013).

54. van Maldergem, L., Wetzburger, C., Verloes, A., Fourneau, C. \& Gillerot, Y. Mental retardation with blepharo-naso-facial abnormalities and hand malformations: a new syndrome? Clin. Genet. 41, 22-24 (1992).

55. Mansour, S. et al. Van Maldergem syndrome: further characterisation and evidence for neuronal migration abnormalities and autosomal recessive inheritance. Eur. J. Hum. Genet. 20, 1024-1031 (2012).

56. Shamseldin, H. et al. RTTN Mutations cause primary microcephaly and primordial dwarfism in humans. Am. J. Hum. Genet. 97, 862-868 (2015).

57. Grandone, A. et al. Expanding the phenotype of RTTN variations: a new family with primary microcephaly, severe growth failure, brain malformations and dermatitis. Clin. Genet. 90, 445-450 (2016).

58. Young, T. R. \& Leamey, C. A. Teneurins: important regulators of neural circuitry. Int. J. Biochem. Cell Biol. 41, 990-993 (2009).

59. da Silva Montenegro, E. M. et al. Meta-analyses support previous and novel autism candidate genes: outcomes of an unexplored Brazilian cohort. Autism Res. 13, 199-206 (2020).

60. Sun, M. W. et al. Coalitional game theory facilitates identification of non-coding variants associated with autism. Biomed. Inf. Insights 11, 1178222619832859 (2019).
61. Narita, A. et al. Clustering by phenotype and genome-wide association study in autism. Transl. Psychiatry 10, 290 (2020).

62. Ballif, B. C. et al. High-resolution array CGH defines critical regions and candidate genes for microcephaly, abnormalities of the corpus callosum, and seizure phenotypes in patients with microdeletions of 1q43q44. Hum. Genet. 131, 145-156 (2012).

63. Luo, A. et al. Maternal interchromosomal insertional translocation leading to 1q43-q44 deletion and duplication in two siblings. Mol. Cytogenet. 11, 24 (2018).

64. Verloes, A. et al. Baraitser-winter cerebrofrontofacial syndrome: delineation of the spectrum in 42 cases. Eur. J. Hum. Genet. 23, 292-301 (2015).

65. Wang, C. C. et al. betalV Spectrinopathies cause profound intellectual disability, congenital hypotonia, and motor axonal neuropathy. Am. J. Hum. Genet. 102, 1158-1168 (2018).

66. Nitarska, J. et al. A functional switch of NuRD chromatin remodeling complex subunits regulates mouse cortical development. Cell Rep. 17, 1683-1698 (2016).

67. Pisansky, M. T. et al. Mice lacking the chromodomain helicase DNA-binding 5 chromatin remodeler display autism-like characteristics. Transl. Psychiatry 7, e1152 (2017).

68. Parenti, I. et al. Missense and truncating variants in CHD5 in a dominant neurodevelopmental disorder with intellectual disability, behavioral disturbances, and epilepsy. Hum. Genet. 140, 1109-1120 (2021).

69. Garfield, A. S. et al. Distinct physiological and behavioural functions for parental alleles of imprinted Grb10. Nature 469, 534-538 (2011).

70. Giovannone, B. et al. Two novel proteins that are linked to insulin-like growth factor (IGF-I) receptors by the Grb10 adapter and modulate IGF-I signaling. J. Biol. Chem. 278, 31564-31573 (2003).

71. Echaniz-Laguna, A. et al. Phenotypic spectrum and incidence of TRPV4 mutations in patients with inherited axonal neuropathy. Neurology 82, 1919-1926 (2014).

72. Sleiman, P. et al. GWAS meta analysis identifies TSNARE1 as a novel Schizophrenia/Bipolar susceptibility locus. Sci. Rep. 3, 3075 (2013)

73. Guan, J., Cai, J. J., Ji, G. \& Sham, P. C. Commonality in dysregulated expression of gene sets in cortical brains of individuals with autism, schizophrenia, and bipolar disorder. Transl. Psychiatry 9, 152 (2019).

74. Park, J. et al. UPF1/SMG7-dependent microRNA-mediated gene regulation. Nat. Commun. 10, 4181 (2019).

75. Jaffrey, S. R. \& Wilkinson, M. F. Nonsense-mediated RNA decay in the brain: emerging modulator of neural development and disease. Nat. Rev. Neurosci. 19, 715-728 (2018).

76. Homs, A. et al. Genetic and epigenetic methylation defects and implication of the ERMN gene in autism spectrum disorders. Transl. Psychiatry 6, e855 (2016).

77. Duman, J. G. et al. The adhesion-GPCR BAI1 regulates synaptogenesis by controlling the recruitment of the Par3/Tiam 1 polarity complex to synaptic sites. J. Neurosci. 33, 6964-6978 (2013).

78. Sund, K. L. et al. Regions of homozygosity identified by SNP microarray analysis aid in the diagnosis of autosomal recessive disease and incidentally detect parental blood relationships. Genet Med. 15, 70-78 (2013).

79. Mirzaa, G. M. et al. De novo and inherited variants in ZNF292 underlie a neurodevelopmental disorder with features of autism spectrum disorder. Genet Med. 22, 538-546 (2020)

80. Schrode, N. et al. Synergistic effects of common schizophrenia risk variants. Nat. Genet. 51, 1475-1485 (2019).

81. Plooster, M. et al. Schizophrenia-linked protein tSNARE1 regulates endosomal trafficking in cortical neurons. J. Neurosci. 41, 9466-9481 (2021).

82. Choi, J. S., Bae, W. Y., Nam, S. \& Jeong, J. W. New targets for Parkinson's disease: adhesion $\mathrm{G}$ protein-coupled receptor B1 is downregulated by AMP-activated protein kinase activation. OMICS 22, 493-501 (2018).

83. Yuen, R. K. C. et al. Whole genome sequencing resource identifies 18 new candidate genes for autism spectrum disorder. Nat. Neurosci. 20, 602-611 (2017).

84. Yuen, R. K. et al. Whole-genome sequencing of quartet families with autism spectrum disorder. Nat. Med. 21, 185-191 (2015).

85. Trost, B. et al. Genome-wide detection of tandem DNA repeats that are expanded in autism. Nature 586, 80-86 (2020).

86. Corbett, R. D. et al. A distributed whole genome sequencing benchmark study. Front. Genet. 11, 612515 (2020).

87. Van der Auwera, G. A. et al. From FastQ data to high confidence variant calls: the Genome Analysis Toolkit best practices pipeline. Curr. Protoc. Bioinforma. 43, 11.10.11-11.10.33 (2013)

88. Ng, P. C. \& Henikoff, S. SIFT: Predicting amino acid changes that affect protein function. Nucleic Acids Res. 31, 3812-3814 (2003).

89. Adzhubei, I. A. et al. A method and server for predicting damaging missense mutations. Nat. Methods 7, 248-249 (2010).

90. Choi, Y. \& Chan, A. P. PROVEAN web server: a tool to predict the functional effect of amino acid substitutions and indels. Bioinformatics 31, 2745-2747 (2015). 
91. Reva, B., Antipin, Y. \& Sander, C. Predicting the functional impact of protein mutations: application to cancer genomics. Nucleic Acids Res. 39, e118 (2011).

92. Rentzsch, P., Witten, D., Cooper, G. M., Shendure, J. \& Kircher, M. CADD: predicting the deleteriousness of variants throughout the human genome. Nucleic Acids Res. 47, D886-D894 (2019).

93. Siepel, A. et al. Evolutionarily conserved elements in vertebrate, insect, worm, and yeast genomes. Genome Res. 15, 1034-1050 (2005).

94. Davydov, E. V. et al. Identifying a high fraction of the human genome to be under selective constraint using GERP. PLoS Comput. Biol. 6, e1001025 (2010).

95. Sievers, F. et al. Fast, scalable generation of high-quality protein multiple sequence alignments using Clustal Omega. Mol. Syst. Biol. 7, 539 (2011).

96. Trost, B. et al. A comprehensive workflow for read depth-based identification of copy-number variation from whole-genome sequence data. Am. J. Hum. Genet. 102, 142-155 (2018).

97. Gel, B. et al. regioneR: an R/Bioconductor package for the association analysis of genomic regions based on permutation tests. Bioinformatics 32, 289-291 (2016).

98. Purcell, S. et al. PLINK: a tool set for whole-genome association and populationbased linkage analyses. Am. J. Hum. Genet. 81, 559-575 (2007).

99. Chang, C. C. et al. Second-generation PLINK: rising to the challenge of larger and richer datasets. Gigascience 4, 7 (2015).

100. Hao, Z. et al. Rldeogram: drawing SVG graphics to visualize and map genomewide data on the idiograms. PeerJ Comput. Sci. 6, e251 (2020).

\section{ACKNOWLEDGEMENTS}

We are grateful to the families for their participation in our study. We also wish to acknowledge the resources of MSSNG (www.mss.ng), Autism Speaks, and The Centre for Applied Genomics at The Hospital for Sick Children, Toronto, Canada. We also thank the participating families for their time and contributions to this database, as well as the generosity of the donors who supported this program. We thank Chelsea Burroughs for assistance in preparing the figures. We thank Jeff MacDonald and Bhooma Thiruvahindrapuram for providing computational support. This work was supported by funding from the University of Texas Southwestern Medical Center and the Walter and Lillian Cantor Foundation to M.H.C., the University of Toronto McLaughlin Centre, The Centre for Applied Genomics, Autism Speaks, and Autism Speaks Canada. S.W.S. holds the Northbridge Chair in Paediatric Research, a joint Hospital-University Chair between the University of Toronto, the Hospital for Sick Children, and the SickKids Foundation. The funders had no role in study design, data collection and analysis, decision to publish, or preparation of the manuscript.

\section{AUTHOR CONTRIBUTIONS}

M.H.C. conceived the study and oversaw the project. I.O.T. and M.H.C. designed experiments. I.O.T., N.L.P., K.K., M.J., and M.H.C. performed experiments and analyzed data. R.K., K.G., P.E., and L.A. referred subjects and reviewed clinical data. A.K. performed, and C.X. supervised, the CNV analysis. J.L.H. and S.W.S coordinated the whole genome sequencing experiments. I.O.T. and M.H.C. wrote the manuscript. All authors participated in reviewing and editing of the manuscript.

\section{COMPETING INTERESTS}

S.W.S. is on the Scientific Advisory Committees of Deep Genomics and Population Bio, and he is an Academic Consultant for King Abdulaziz University. S.W.S. is Editorin-Chief of npj Genomic Medicine. Other authors declare no competing interests.

\section{ADDITIONAL INFORMATION}

Supplementary information The online version contains supplementary material available at https://doi.org/10.1038/s41525-022-00284-2.

Correspondence and requests for materials should be addressed to Maria $\mathrm{H}$. Chahrour.

Reprints and permission information is available at http://www.nature.com/ reprints

Publisher's note Springer Nature remains neutral with regard to jurisdictional claims in published maps and institutional affiliations.

(i) Open Access This article is licensed under a Creative Commons Attribution 4.0 International License, which permits use, sharing, adaptation, distribution and reproduction in any medium or format, as long as you give appropriate credit to the original author(s) and the source, provide a link to the Creative Commons license, and indicate if changes were made. The images or other third party material in this article are included in the article's Creative Commons license, unless indicated otherwise in a credit line to the material. If material is not included in the article's Creative Commons license and your intended use is not permitted by statutory regulation or exceeds the permitted use, you will need to obtain permission directly from the copyright holder. To view a copy of this license, visit http://creativecommons. org/licenses/by/4.0/.

(c) The Author(s) 2022 